Journal of Computer Science 7 (2): 291-297, 2011

ISSN 1549-3636

(C) 2011 Science Publications

\title{
Implementation of Computer Aided Diagnosis System Based on Parallel Approach of Ant Based Medical Image Segmentation
}

\author{
${ }^{1}$ Jayabalan Jaya and ${ }^{2}$ Keppanagowder Thanushkodi \\ ${ }^{1}$ Department of Electronics and Communication Engineering, \\ Hindusthan Institute of Technology, Coimbatore, Tamil Nadu, India \\ 2.Director of Akshaya College of Engineering and Technology, Coimbatore, Tamil Nadu, India
}

\begin{abstract}
Problem statement: The aim of this study is to develop Computer Aided Diagnosis (CAD) system for the detection of brain tumor by using parallel implementation of ACO system for medical image segmentation applications due to the rapid execution for obtaining and extracting the Region of Interest (ROI) from the images for diagnostic purposes in medical field. Approach: For ROI segmentation, metaheuristic based Parallel Ant colony Optimization (PACO) approach has been implemented. The system has been simulated in the Mat lab for the parallel processing, using the master slave approach and information exchange. The scheme is tested up to 10 real time MRI brain images. Here parallelism is inherent in program loops, which focused on performing searching operation in parallel. Results: The computational results shows that parallel ACO systems uses the concept of the parallelization approach enabled the utilization of the intensity similarity measurement technique because of the capability of parallel processing. Conclusion: Medical image segmentation and detection at the early stage played vital roles for many health-related applications such as medical diagnostics, drug evaluation, medical research, training and teaching. Due to the rapid progress in the technologies for segmenting digital images for diagnostic purposes in medical field parallel Ant based CAD system are technologically feasible for Medical Domain which will certainly reduce the mortality rate.
\end{abstract}

Key words: Computer Aided Diagnosis (CAD), Brain tissue, CAD system, skeletal systems, interpret brain images, Parallel Ant colony Optimization (PACO), Region of Interest (ROI), Non Rigid Registration Segmentation (NRRS), Weighted Median (WM)

\section{INTRODUCTION}

The aim of this study is to develop Computer Aided Diagnosis (CAD) system for the detection of brain tumor by using Metaheuristic Algorithms. Brain tissue has a complex structure and its segmentation is an important step for deriving the computerized anatomical atlases as well as pre and intra operative guidance for therapeutic intervention .The accurate quantification of disease patterns in medical images allows the radiologists to track the status of the disease. Image analysis is still performed manually which is often a difficult and time-consuming task. As a result, there is an increasing need for computerized image analysis to facilitate image based diagnosis. Many investigators have carried out basic studies and clinical applications toward the development of modern computerized schemes called CAD system for detection and characterization of lesions in images such as brain, chest, colon, breast, liver, kidney and the vascular and skeletal systems. The early detection is the most effective way to reduce mortality.

Most of the radiologists achieve this goal with the process of image perception to recognize the unique image pattern to identify the relationship between the perceived patterns and the possible diagnosis. But both detection and characterization processes depend heavily on the radiologists' empirical knowledge, memory, intuition and diligence. So, there are chances for well documented errors and variations in the human interpretation of clinical images. Indeed, the estimates indicate that between 10 and $30 \%$ of tumors are missed by the radiologists during the routine screening the intension of the intelligent system is not to replace the radiologists but to provide them with a second opinion on a lesion diagnosis to achieve high accuracy and save human lives.

The early detection being a key factor in producing successful results, it is of permanent importance to

Corresponding Author: J. Jaya, Electronics and Communication Engaging, Hindusthan Institute of Technology, Coimbatore, Tamil Nadu, India Tel: 9176662859 
improve the ability of identifying tumors at the earliest stage. It is difficult to interpret brain images, as the probability of encountering an abnormality is low and the information of the patient is limited. It takes a trained radiologist to segment the suspicious region without missing any abnormality. In the development of CAD systems, it is the computer that essentially acts as a second reader and so a large number of cases can be examined without an increase in cost. Furthermore, it can help to get better sensitivity, cost effectiveness and less time-consumption.

The CAD system can provide the valuable outlook and accuracy of earlier brain tumor detection. The two key steps involved in the implementation of CAD system are segmentation and classification of suspicious regions (Jaya and Thanushkodi, 2009a). Segmentation algorithm has two stages: (i) Bilateral registration segmentation (ii) Single image segmentation. Further the classification is based on pixel similarity index. The detection of brain tumor using CAD system is performed in four phases namely: Image Acquisition and pre-processing, enhancement, segmentation and classification.

\section{MATERIALS AND METHODS}

Image acquisition and pre-processing: Image preprocessing indicates that the same tissue type may have a different scale of signal intensities for different images. It depends on the modality and corrects the system irregularities such as differential light detection efficiency, dead pixels or dark noise. The preprocessing aspects are surveyed and analyzed in the following first section.

Nerve fiber tracking method was proposed for preprocessing of brain images (Metaxas et al., 2006). It required the alignment of axons and mainly suited for well defined, less distorted and diffusion tensor images. The principal component is designed (Parker and Feng, 2002). to minimize the artifacts present in the dataset. A new method on statistical parametric mapping to confer robustness to areas of abnormality was introduced (Schmidt et al., 2005). Fourier transformation technique was used to reduce the radiometric differences. But this method did not support for additive intensity noise field. Content based model (Udupa et al., 2002) was used. However this method concentrated just to remove the baselines and this method suited to remove low frequency flat distortion noise. The histogram based technique is to separate the brain image by removing the residual fragments (Zheng and Chellappa, 1993) Moreover this method was applicable for low field MRI brain images.
At present the pre-processing is done in single stage mainly concentrating the removal of the film artifacts alone. These drawbacks in the existing works are overcome by our implemented new tracking algorithm (Jaya and Thanushkodi, 2009b) in which preprocessing is done in two stages. The pixel intensities of the labels in the MRI scan and tumor seem to be equal but they are actually not. Hence there is a need for removing film artifacts. In this study, an efficient tracking algorithm is proposed for this purpose. In MRI brain image, the tumor region and the non- brain portion are viewed as a white matter which causes unnecessary confusion for the further process. Due care is taken to remove the non-brain tissues from the image using skull-stripping algorithm.

Enhancement: The pre-processed MRI brain image contains a high intensity salt and pepper noise which appears due to the presence of gray scale variations in the image which is removed by applying suitable filters and performing normalization. Hence the objective of enhancement is de-noising the high frequency components. Adaptive filter was implemented (Tsai et al., 1995) to remove local noisy fluctuations and the outlines of the bone and soft tissues. Gabor filter (Metaxas et al., 2006) was applied to remove the tagging lines and enhance the tag-patterned regions in the image. It suited particularly for texture representation and discrimination. Anisotropic diffusion filter (Amit, 1997) for the registered images was used. Gadolinium compounds as intravenous MRI contrast agent to enhance the brain images. It was applicable only at room temperature as it possesses ferromagnetic Curie point (17 degree centigarde) and at high temperature it acted with unusual metallurgic property. Prewitt edge-finding filter to enhance the image edges robustly. A new method based on morphological operations was proposed for automatic detection of lesions (Bai et al., 2003) for removing backgrounds from brain images. Gaussian filters were also used to enhance the image and make the image gradients stronger.

These drawbacks are minimized by our implemented Weighted Median (WM) filters (Jaya and Thanushkodi, 2009a) for de-noising purpose. WM filtering is an enhancement technique for removing noise without significantly reducing the sharpness of the image. WM filter reduces noise in an image by preserving useful details. These filters have the robustness and edge preserving capability of the classical median filter. WM filters belong to the broad class of nonlinear filters. It considers each pixel value with the 
$\mathrm{WM}$ of the neighboring pixel value. The WM filter is a variation of the median filter that incorporates spatial information of the pixels while computing the median value. $A W M$ value $W(x, y)$ is calculated using Eq. 1:

$\mathrm{W}(\mathrm{x}, \mathrm{y})=\operatorname{median}\{\mathrm{w} 1 \times \mathrm{x} 1 \ldots \ldots \ldots \mathrm{W} \mathrm{n} \times \mathrm{x} \mathrm{n}\}$

where, $\mathrm{x} 1 \ldots \ldots \mathrm{x} \mathrm{n}$ are the intensity values inside a sliding window centered at $(\mathrm{x}, \mathrm{y})$ and $\mathrm{w} \times \mathrm{n}$ denotes replication of $\mathrm{x}, \mathrm{w}$ times.

In WM filter, the weights to the pixels are given as follows: If the intensity value is less than 50 , a weight 0.1 is multiplied with the intensity value, else if the intensity value ranges from $51-100$, a weight of 0.2 is multiplied with the intensity value and if the intensity value ranges from 101-255-a weight of 0.3 is multiplied with the intensity value. The median value is calculated by sorting the resultant weighted pixel values in ascending order.

WM filter is capable of removing salt and pepper noise from MRI without disturbing the edges. Here, salt corresponds to the maximum gray value (white) and pepper corresponds to the minimum gray value (black).In this enhancement stage, the WM filter is applied for each pixel of $3 \times 3,5 \times 5$ and $7 \times 7$ sliding window of neighborhood pixels. The mean gray value of foreground and mean gray value of background are noted and hence the contrast value is calculated using Eq. 2:

$C=(f-b) /(f+b)$

Where:

$\mathrm{F}=$ The mean gray -level value of the foreground

$\mathrm{B}=$ The mean gray-level value of the background

Finally the performance evaluation of various filters is compared in terms of Signal to Noise Ratio (SNR) value. The snap shot of implemented preprocessing and enhancement stage is given in the following Fig. 1.

Bilateral Registration Segmentation: Bilateral segmentation is a straightforward evaluation method that is commonly used for comparing the corresponding MRI brain images to determine the ROI in the image. Points contour and curves based suggested registration technique is suggested (Amit, 1997). The operator can exactly extract the features from the images with semiautomatic extraction method. This method is efficient but works well only on cases where the contour information is well preserved. An elegant method called phase correlation (De Castro and Morandi, 1987) is introduced. However, when the overlapping area between images is small, their method becomes unreliable. The method for determining the rotation parameter was proposed (Zheng and Chellappa, 1993), this method used a Lumberton method to model an image. Rahmatizadeh et al. (2009) used the ABR for the segmentation. By adopting the method proposed by (Manjunath and Mitra, 1995; Manjunath et al., 1992) a number of feature points extracted from the image pair and these feature points were matched by using hierarchical image structure. Hashim and Marghany (2009) applied Robust of Doppler Centroid for Mapping Sea Surface. Shareha et al. (2009) used Ontology Alignment. But this approach failed when false matches emerge.

The drawback of these approaches is overcome by our implemented registration techniques (Jaya and Thanushkodi, 2010) which are based on the pixel intensity values. Bilateral registration segmentation is done in two phases. In the first phase segmentation is done by Rigid Registration Segmentation (RRS) technique and in the second phase segmentation is done by Non Rigid Registration Segmentation (NRRS) technique. Then the suspicious region is extracted by using an efficient metaheuristic Genetic Algorithm (GA).

In our previously implemented RRS technique, statistical similarity measures such as contrast checking, Sum of Squared Difference (SSD), calculation of white cells and point mapping are performed. This statistical analysis is used to localize and make inferences about pixel intensity differences. The output from the RRS technique shows that the high intensity pixel regions significantly differ with respect to the normal image. On comparing the smoothed MRI target images with the normal image, the tumor portion can be segmented. In this study smoothing the boundary of ROI is done by GA. The Fig. 2 explains snap shot of implemented RRS with GA segmentation.

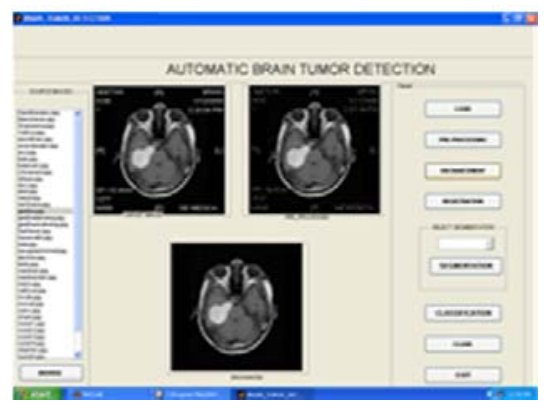

Fig.1: Screen shots of pre-processing and Enhancement of corresponding acquired image 


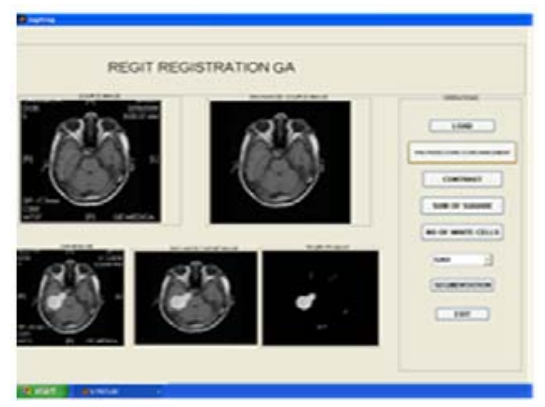

Fig. 2: Segmentation Screen Shot of Corresponding to RRS with GA

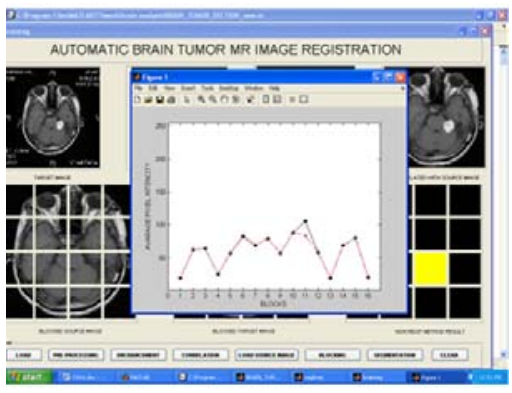

Fig. 3: Segmented screen shot of bilateral images corresponding NRRS with GA technique

In the second phase segmentation by the previously implemented NRRS technique is block based (area based) approach in which the normal image and the target images are divided into block of size $64 \times 64$ and compared with each blocks. The block based method adopts sliding window to determine a matched location using the correlation technique. Normalized crosscorrelation is the most commonly used measure which is more robust than the feature-based. Figure 3 explains the Segmented Screen Shot of Bilateral Images Corresponding NRRS with GA Technique. In the bilateral registration, segmentation is performed by comparing with the reference image and therefore it was not provided good classification accuracy. Therefore the single image segmentation is proposed in this study.

Parallel ant colony optimization: In this study single image segmentation through PACO is proposed to overcome the drawback of bilateral registration segmentation. In ACO, real ants are capable of finding the shortest path from a food source to the nest without using visual cues. In many ant species, ants walking to and from a food source deposit a substance called Pheromone on the ground. Other ants perceive the presence of pheromone and tend to follow the paths where pheromone concentration is higher. Through this mechanism, ants are able to transport food to their nest in a remarkably effective way (Devos et al., 2004; Dorigo et al., 1996). Wu et al. (2010) has implemented ACO for CDMA multiuser detector,he thoroughly investigated the pheromone laying and following behavior of ants that the higher the pheromone concentration found on a particular path, the higher is the probability to follow that path. This elementary behavior of real ants can be used to obtain optimum value from a population. Nermine and Elsayed (2009) have implemented Honey Bee Venon algorithm for immune system. The ACO algorithm is implemented to select the optimum label and the pixels having this optimum label are extracted from the original brain image to form the segmented image. This elementary behavior of real ants can be used to obtain optimum value from a population. The ACO algorithm is implemented to select the optimum label and the pixels having this optimum label are extracted from the original brain image to form the segmented image.

PACO is a parallel implementation of ACO where ants do their study simultaneously on different processing units. This intuitively provides improved performance and speeds up the searching process by exchanging information about the solutions they found. In this proposed PACO, master-slave and information exchange approaches are combined to improve the result of image segmentation. The suspicious region is segmented using PACO and performance evaluations are evaluated.

PACO independently executes the sequential algorithm on M-1 parallel sub colonies. Parallel runs have no communication overhead. They are useful in randomized algorithms. In case of parallel independent runs, the best solution of the $M$ runs is taken as the final solution. To speed up the searching process the masterslave approach is introduced in the proposed study. One master colony is used to update the main data structures for the ACO algorithm, constructing initial solutions for the local search algorithms and sending the solutions to other sub colonies which improve them by local search. The master collects these locally optimal solutions and in case a sufficient number of such solutions have been arrived at, it updates the trail matrix before constructing more solutions. The master is responsible only for spawning the slaves and prunes them whenever a slave returns the optimal to the master. In all the processing, the local search and the pheromone matrix update are done by the slaves. Slaves also exchange solutions independently from the master to decrease the overhead of the communication with the master. The master spawns a number of slaves and each slave finds the best solution and sends it back to the master. During this operation, each slave periodically exchanges information with its neighbor. Each slave uses this 
information to update its pheromone matrix. The best solution is determined by the tour length. Each subcolony chooses the tour with the minimum length as its best and reports it to the master program at the end of its search. The master program finds the best solution it received from its slaves and presents this as the proposed solution.

In this approach, each colony is given a valid IDs and they select their partners so that the slaves with even IDs exchange their information with their successors and those with odd IDs exchange their local optimal with their predecessors. Therefore, each colony exchanges information with the same colony at the end of each time interval. Slaves exchange three parameters: their pheromone matrices, the iteration length and the best iteration obtained so far. For each colony, the best ant represents its sub colony and its best iteration and length are sent to its partner colony.

Whenever the master receives the optimal solution, it multicasts to all slaves that this solution has been found and the slaves consequently prune themselves. This reduces the time wasted in other slaves who would not know otherwise that another slave has found the optimal solution.

The fundamental principle of PACO is to divide $\mathrm{K}$ ants into $\mathrm{M}$ sub colonies, so that the number of ants per each sub ant colony is the total number of ants divided by the number of sub colonies. In the algorithm designing, each colony is treated as an independent processor and then the ant colony can search the best solution independently. In order to avoid the local optimization in some colonies when the ant is doing the job, the other sub colonies should carry out the information exchange with each other in the chosen fixed time interval condition so that the execution time is reduced very much. The above Fig. 4 shows the segmented screen shot of MRI image using single image segmentation by adopting PACO technique.

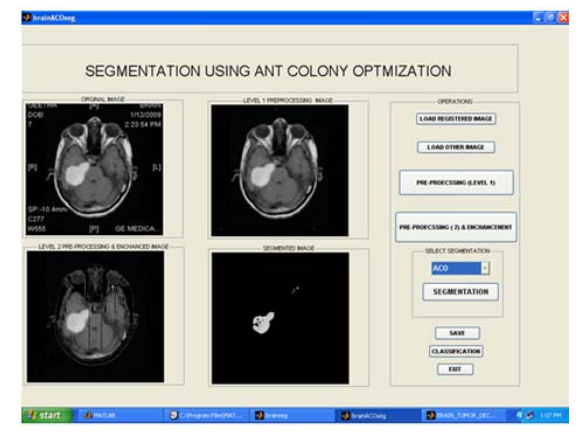

Fig. 4: Segmented screen shot of single image corresponding PACO Technique

\section{RESULTS}

Modifications in ACO: In ACO each individual ant constructs a part of the solution using an artificial pheromone which reflects its experience accumulated while solving the problem. But in the proposed PACO approach, totally $\mathrm{M}$ colonies are considered in which M-1 colonies are treated as slaves and one colony is assigned for master. Each colonies visit all the pixels without revisit. Initially, the pheromone value for all the colonies is initialized and the posterior energy values are computed. Finally each slave colony yields global optimum value and the master colony system also yields global optimum value. Therefore M-1 slave colonies produce M-1 optimum values. These values are compared and the highest global optimum value from slave colonies is computed and compared with the master global value. If the values of the slave colonies are less than the master value then the values are discarded otherwise the values are interchanged or swapped. This optimum value is treated as adaptive threshold value. In the MRI image, the pixels having lower intensity values than the threshold value are changed to zero. The entire procedure is repeated for number of times to obtain the more accurate value.

The time taken to find the optimal solution using PACO is much shorter than that of using the sequential ACO. The sequential ACO runs $\mathrm{M}$ tries on a single colony whereas PACO runs single try on $\mathrm{M}$ colonies.

Equation (3) is the PACO equation which is the modified ACO equation.

The Fig. 5 demonstrates the difference in time taken by sequential ACO and PACO to find the optimal solution.

Table 1: Performance Analysis of Proposed PACO with the existing segmentation techniques

\begin{tabular}{lllll}
\hline Methods & $\begin{array}{l}\text { Neighborhood } \\
\text { pixel values }\end{array}$ & $\begin{array}{l}\text { Adaptive } \\
\text { threshold }\end{array}$ & $\begin{array}{l}\text { Number of } \\
\text { segmented } \\
\text { pixels }\end{array}$ & $\begin{array}{l}\text { Execution } \\
\text { time } \\
(\mathrm{sec})\end{array}$ \\
\hline HMRF-FCM & $6 \times 6$ & 190 & 1223 & 100.03 \\
ACO-FCM & $3 \times 3$ & 187 & 1800 & 27.09 \\
Metaheuristic GA & $3 \times 3$ & 185 & 1389 & 14.50 \\
Proposed PACO & $3 \times 3$ & 170 & 1026 & 10.00 \\
\hline
\end{tabular}

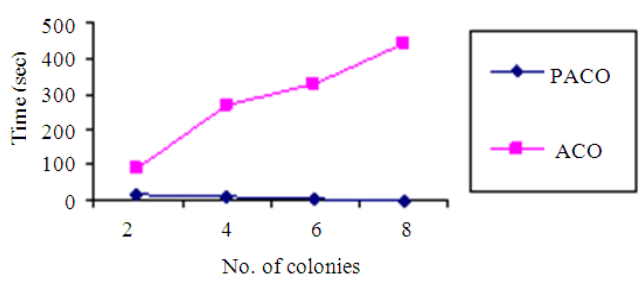

Fig. 5: Time taken to find optimum solution by ACO and PACO 


\section{DISCUSSION}

The run time of PACO for finding optimal solution is much shorter than that of using the sequential ACO. The following changes have been done in the sequential ACO.

\section{Steps for Master program in PACO:}

Step 1: Initialize number of processes $\mathrm{Np}$.

Step 2: Start Timer

Step 3: Spawn Np processes

Step 4: Multicast to all slave processes Np and the task IDs of all slaves.

Step 5: For each slave do send a number between 0 and $\mathrm{Np}$ that identifies the task inside the program till all slaves send back solution.

Step 6: If a slave returns an optimum solution that is better than any solution received before, multicast this tour length to all the slaves and stop the timer.

Step7: Get elapsed time and best solution received.

\section{Steps for Slave program:}

Step 1: Get Np and task IDs of all slaves from the master

Step 2: Initialize pheromone matrices.

Step 3: For each try, check the reachability of termination condition (maximum allowed time and optimal solution found, if the master received a new optimal solutions, prune this slave and update pheromone matrix)

Step 4: Identify neighbor for information exchange and send to neighbor the best tour found, its length and the pheromone matrix.

Step 5: Update pheromone matrix using information received from neighbor.

Step 6: Send to master the best solution found.

After information exchanging has taken place, each slave updates its pheromone matrix according to the following Eq. 3:

$$
\tau \text { new }=\rho * \tau \text { old }+(\text { fit_m/(fit_m+fit_h }))^{*} \text { cold }
$$$$
\mathrm{m}(\mathrm{t})+(\text { fit_h/(fit_m+fit_h}))^{*} \tau \text { old } \overline{\mathrm{h}}(\mathrm{t})
$$

Where:

$$
\begin{array}{ll}
\mathrm{M} & =\text { Master colony } \\
\mathrm{H} & =\text { Slave of the colony } \\
\tau \mathrm{m}(\mathrm{t}+1) & =\text { New pheromone element after update } \\
\tau \mathrm{m}(\mathrm{t}) & =\text { Pheromone element before update } \\
\text { fit } & =\text { Fitness of the processor's best iteration }
\end{array}
$$

\section{CONCLUSION}

The advantage of our proposed approach is, all the slaves need not send and compare its value to the master every time. But instead the slaves exchange their findings among themselves by information exchange mode and select the local optimal value. Then this local optimal value is compared with the master optimal value and thus global optimal value is found. Hence the runtime complexity can be enormously reduced. Once the optimal value is predicted and then the ROI is found based on PACO. The classification efficiency obtained by PACO is $98.79 \%$ which is 2 times more than that of the existing methods. Table 1 shows the performance analysis of proposed PACO with the existing segmentation techniques.

\section{REFERENCES}

Amit, Y., 1997. Graphical shape templates for automatic anatomy detection with applications to MRI brain scans. IEEE Trans. Med. Imag., 16: 2840. DOI: $10.1109 / 42.552053$

Bai, X., J.S. Jin and D. Feng, 2003. Segmentation-based multilayer diagnosis lossless medical image compression. Proceeding of the Australian Computer Society, Pan Sydney Area Workshop on Visual Information Processing, (VIP'03), Australian Computer Society, Inc. Darlinghurst, Australia, pp: 9-14.

De Castro, E. and C. Morandi, 1987. Registration of translated and rotated images using finite Fourier transform. IEEE Trans. Pattern Anal. Machine Intell., $\quad 9$ : 700-703. DOI: 10.1109/TPAMI.1987.4767966

Devos, A., L. Lukas, A.W. Simonetti, J.A.K. Suykens and L. Vanhamme et al., 2004. Does the combination of magnetic resonance imaging and spectroscopic imaging improve the classification of brain tumours? Proceeding of the 26th Annual International Conference on Engineering in Medicine and Biology Society, Sept. 1-5, San Francisco, CA, pp: 407-410. DOI: 10.1109/IEMBS.2004.1403180

Dorigo, M., V. Maniezzo and A. Colorni, 1996. Ant system: Optimization by a colony of cooperating agents. IEEE Trans. Syst. Man Cybernetics, 26: 29-41. DOI: $10.1109 / 3477.484436$

Hashim, M. and M. Marghany, 2009. Robust of doppler centroid for mapping sea surface current by using radar satellite data. Am. J. Eng. Applied Sci., 2: 781-788. DOI: 10.3844/ajeassp.2009.781.788

Jaya, J. and K. Thanushkodi, 2009a.Tracking algorithm for de-noising of MR brain images. Int. J. Comput. Sci. Network Security, 9: 262-267. 
Jaya. J. and K. Thanushkodi, 2009b. Structural modeling and analysis of Computer Aided Diagnosis (CAD) system: A graph theoretic approach. Int. J. Comp. Sci. Appl., 2: 5-8.

Jaya. J. and K. Thanushkodi, 2010.Exploration on selection of medical images employing new transformation model. Int. J. Comput. Sci. Iss., 7: 43-48.

Manjunath, B.S. and S.K. Mitra, 1995. A contour-based approach to multisensor image registration. IEEE Trans. Image Process., 4: 320-334. DOI: 10.1109/83.366480

Manjunath, B.S., R. Chellappa and C. Malsburg, 1992. A feature based approach to face recognition. Proceeding of the IEEE Conference on Computer Vision Pattern Recognition, June 15-18, Champaign, Il, USA., pp: 373-378. DOI: 10.1109/CVPR.1992.223162

Metaxas, D.N., Z. Qian, X. Huang, R. Huang and T. Chen et al., 2006. Hybrid deformable models for medical segmentation and registration. Proceeding of the 9th International Conference on Control, Automation, Robotics and Vision, Dec. 5-8, Singapore, $\quad$ pp: 1-6. DOI: 10.1109/ICARCV.2006.345077

Nermine, K.M.S. and A.A. Elsayed, 2009. Immunological effects of honey bee venom in mice with intracerebral candidiasis. J. Med. Sci., 9: 227-233. DOI: $10.3923 /$ jms.2009.227.233

Parker, B. and D.D. Feng, 2002. Variational Segmentation and PCA Applied to dynamic PET analysis. Proceeding of the Pan-Sydney Workshop on Visualisation, (PSWV'02), Australian Computer Society, Inc. Darlinghurst, Australia, pp: 89-91.
Rahmatizadeh, Sh., H. Shah-Hosseini and H. Torkaman, 2009. The ant-bee routing algorithm: A new agent based nature-inspired routing algorithm. J. Applied Sci., 9: 983-987. DOI: 10.3923/jas.2009.983.987

Schmidt, M., I. Levner, R. Greiner, A. Murtha and A. Bistritz, 2005. Segmenting brain tumors using alignment-based features. Proceeding of the 4th International Conference on Machine Learning an Applications, (ICMLA'05), IEEE Computer Society Washington, DC, USA., pp: 215-220. DOI: 10.1109/ICMLA.2005.56

Shareha, A.A.A., M. Rajeswari and D. Ramachandram, 2009. Multimodal integration (image and text) using ontology alignment. Am. J. Applied Sci., 6: 1217-1224. DOI: 10.3844/ajassp.2009.1217.1224

Tsai, C., B.S. Manjunath and R. Jagadeesan, 1995.Automated Segmentation of brain MR images. Patt. Recognition, 28: 1825-1837. doi: 10.1016/0031-3203(95)00047-X

Udupa, J.K., V.R. Lablane, H. Schmidt, C. Lmielinska and P.K. Saha et al., 2002. Methodology for evaluating image-segmentation algorithms. Proceeding of the IEEE International Conference on Medical Imaging and Image Processing, Feb. 25-25, San Diego, CA, USA, pp: 266-266. DOI: $10.1117 / 12.467166$

Wu, Z., Y. Kuang, N. Zhao and Y. Zhao, 2010. A hybrid CDMA multiuser detector with ant colony optimization and code filtering system. Inform. Technol. J.., 9: 818-824.

Zheng, Q. and R. Chellappa,1993. A computational vision approach to image registration. IEEE Trans. Image Process., 2: 311-326. DOI: 10.1109/83.236535 